'Vicerrectoría de Investigación y Postgrado, Universidad Católica del Maule. Talca, Chile. ${ }^{2}$ Departamento de Ciencias de la Actividad Física, Pedagogía en Educación Física, Facultad de Educación, Universidad Católica del Maule. Talca, Chile. ${ }^{3}$ Departamento de Ciencias de la Actividad Física, Universidad de Los Lagos. Osorno, Chile.

${ }^{4}$ Instituto de Actividad Física y Salud, Universidad Autónoma de Chile. Temuco, Chile.

${ }^{5}$ Recerca de l'Educació Física i I'Esport (EDUFISES), Universitat de Barcelona, España. ${ }^{6}$ ELHOC Research Group Epidemiology of Lifestyle and Health Outcomes in Chile. aprofesor de Educación Física. ${ }^{\mathrm{b}} \mathrm{MsC}$.

'Programa de Doctorado en Educación Universidad Academia del Humanismo Cristiano, Chile.

dPrograma de Doctorado en Ciencias de la Actividad Física,

Facultad de Ciencias de la Educación, Universidad Católica del Maule. Talca, Chile. ePhD.

Conflicto de interés: no hay conflicto de intereses. Financiamiento: sin financiamiento.

Recibido el 31 de enero de 2018, aceptado el 4 de junio de 2018.

Correspondencia a: Rodrigo Ramírez-Campillo Universidad de Los Lagos, Campus Chuyaca, Avenida Fuchslocher $n^{\circ} 1305$, Osorno, Chile. r.ramirez@ulagos.cl

\section{Ecuación para predecir el consumo máximo de oxígeno a partir de la prueba de caminata de seis minutos en jóvenes sanos}

\author{
JAIME VÁSQUEZ-GÓMEZ2,5,6,a,b,e MARCELO CASTILLO-RETAMAL ${ }^{2, a, b, e}$,

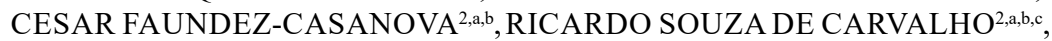 \\ RODRIGO RAMÍREZ-CAMPILLO ${ }^{3, a, b, e}$, PABLO VALDÉS-BADILLA ${ }^{4, a, b, d}$
}

\section{An equation to predict maximum oxygen consumption from the six-minute walk test in healthy young adults}

Background: The six-minute walk test (SMWT) is an easy-to-use test that measures walking distance. Aim: To elaborate an equation to estimate the maximum oxygen consumption ( $\mathrm{VO}_{2}$ max) using the results of the SMWT. Material and Methods: Forty men and 40 women aged $22.5 \pm 2$ years, underwent a $S M W T$ to measure the total walked distance and the recovery heart rate (RhR) Also, $V O$, max was estimated from the maximal workload achieved in a cyclo-ergometer using the Storer test. A multivariate regression analysis resulted in a prediction equation that was validated with distributional assumptions of normality, independence and homoscedasticity. The limits of concordance of the predictive model were checked with the Bland-Altman diagram. Results: Body mass index (BMI), sex, $\mathrm{RhR}$ and total walked distance explained $\mathrm{VO}_{2}$ max variance by 3.4, 73.1, 17.9 and $32.8 \%$, respectively. The prediction equation achieved was $\mathrm{VO}_{2}$ max $\left(\mathrm{ml}_{\mathrm{min}}{ }^{-1}\right)=-3672.585+(966.472 \times$ Sex [1: female, 2: male $])+\left(-18.492 \times R h R\left[\right.\right.$ beats.minute $\left.\left.{ }^{-1}\right]\right)+(9.191 \times$ Distance $[m])+$ $(87.707 \times B M I)$. The $R^{2}$ of the equation was $0.91(p<0.01)$. Conclusions: This equation predicts $\mathrm{VO}_{2}$ max in Chilean university students according to sex, BMI, cardiovascular response and performance in the SMWT.

(Rev Med Chile 2018; 146: 830-838)

Key words: Cardiorespiratory Fitness; Exercise Test; Oxygen Consumption; Walking.
L a prueba de caminata de seis minutos (P6CM) no es invasiva, es estandarizada fácil de realizar ${ }^{1,2}$, de esfuerzo submáximo y se mide la distancia recorrida ${ }^{3,4}$. Se ha documentado que la distancia es información limitada de la capacidad funcional siendo necesario un modelo predictivo del $\mathrm{VO}_{2}$ máx ${ }^{5}$. De hecho la distancia se ha relacionado con la capacidad de consumir oxígeno encontrándose relaciones significativas entre estas variables en personas con patologías, pudiendo ser distinto en personas saludables ${ }^{6}$. De todos modos, la correlación entre la distancia y $\mathrm{VO}_{2}$ máx. oscila entre 0,34 y $0,74^{4}$. También se ha asociado el $\mathrm{VO}_{2}$ máx. con el $\mathrm{IMC}^{2,5,7}$ y con el peso corporal $^{8}$ como variables potencialmente predictoras.

En la literatura científica encontramos investigaciones que generan ecuaciones para predecir 
el $\mathrm{VO}_{2}$ máx. a partir de la PC6M, en personas con insuficiencia cardíaca ${ }^{9}$, niños obesos ${ }^{7}$, adultos mayores saludables ${ }^{10}$, personas con disfunción sistólica ${ }^{11}$ y con enfermedad cardíaca ${ }^{5}$. Estos últimos autores utilizan un test cardiopulmonar de criterio que estima de manera indirecta el $\mathrm{VO}_{2}$ máx. para elaborar una ecuación de predicción para la PC6M.

Si bien existen modelos predictivos para diferentes muestras de la población, es difícil encontrar para universitarios, sobre todo en Chile a excepción de la ecuación para estudiantes de educación física ${ }^{12}$, por lo que es pertinente cubrir esta necesidad y aportar una fórmula para la predicción de la capacidad cardiopulmonar, ya que las elaboradas en otros contextos responden a características corporales y funcionales distintas de las personas, propias de las diferencias genéticas y ambientales entre países. En esta línea, la investigación se justifica ya que el modelo pretende estimar el $\mathrm{VO}_{2}$ máx., variable que no incorpora la PC6M originalmente y para ello se considerarán variables fáciles de medir y que no requieren un material de elevado coste económico lo que facilitaría su aplicación por los profesionales. Por tanto, el objetivo fue elaborar una ecuación para estimar el $\mathrm{VO}_{2}$ máx. a partir de la PC6M para estudiantes universitarios chilenos de ambos sexos.

\section{Materiales y Métodos}

Realizaron las pruebas universitarios de ambos sexos de diferentes carreras (excepto educación física) de una universidad del sur de Chile. Participaron 40 mujeres y 40 hombres (Tabla 1) tras excluir a 11 estudiantes para fines del análisis estadístico que no realizaron adecuadamente los test: no cumplieron el criterio de llegar a la fatiga física y aproximarse al 100\% de la frecuencia cardíaca máxima estimada según la edad (200-edad) para claudicar el test de Storer. Se invitó a los estudiantes: a) no realizar ejercicio físico $48 \mathrm{~h}$ antes de las mediciones; b) no ingerir alcohol; c) no ingerir bebidas que alteren la frecuencia cardíaca (FC) antes de las pruebas y c) consumir una comida no pesada dos horas antes. Los participantes firmaron un consentimiento informado guiado por las directrices sobre la investigación con humanos por la declaración de Helsinski y estuvo respaldado por el Comité de Ética de la Universidad Católica del Maule, Chile ( $\mathrm{N}^{\circ}$ 87/2017).
La PC6M consiste en recorrer la mayor distancia posible y se aplicó en un pasillo de 30 metros $^{13}$ parcializado cada un metro. Se midió la FC con un monitor marca Polar modelo V800 (Finlandia) durante el test y en una recuperación de tres minutos bípeda y estacionaria, también se midió la percepción subjetiva del esfuerzo (PSE) al finalizar ${ }^{14}$ y la distancia.

La prueba del tipo "gold standard" para evaluación del $\mathrm{VO}_{2}$ máx. ${ }^{2}$ fue el test de Storer ${ }^{15}$ realizada en un cicloergómetro marca Monark modelo Ergomedic 282E (Suecia) y consistió en pedalear a 60 revoluciones por minuto según un metrónomo iniciándose con carga de 15 vatios aumentando a razón de 15 vatios cada minuto. Este es un test previamente validado, de manera que como alternativa a la medición directa se puede utilizar. ${ }^{4}$ Se midió la FC, la PSE al finalizar, la última carga en vatios soportada y se estimó el $\mathrm{VO}_{2}$ máx. $\left(\mathrm{ml}^{-m_{i n}}{ }^{-1}\right)$ :

$$
\begin{aligned}
\text { Hombres }= & (10,51 \times \mathrm{W})+(6,35 \times \mathrm{kg})- \\
& (10,49 \times \mathrm{edad})+519,3 \mathrm{ml} \cdot \mathrm{min}^{-1} \\
\text { Mujeres }= & (9,39 \times \mathrm{W})+(7,7 \times \mathrm{kg})-(5,88 \times \text { edad }) \\
& +136,7 \mathrm{ml} \cdot \mathrm{min}^{-1}
\end{aligned}
$$

Donde "W" es la última carga en vatios soportada, "kg" es el peso corporal y la "edad" en años.

Se realizó solamente una sesión en la cual se aplicó las dos pruebas en el Laboratorio de Rendimiento Humano de la Universidad Católica del Maule. Primero se recogió los consentimientos informados en papel, luego se tomaron datos básicos (fecha de nacimiento y evaluación, año ingreso a la universidad, carrera universitaria), presión arterial sistólica y diastólica, y la FC de reposo con un monitor de presión arterial automático marca OMRON modelo BP760 serie 7 (Japón), la saturación de oxígeno con oxímetro de pulso marca Carewell modelo F1 (China) y el peso corporal y estatura sin calzado y con ropa ligera con una báscula mecánica con estadímetro marca DETECTO modelo 3P7044 de capacidad de $140 \mathrm{~kg}$ (EE.UU.). Posteriormente se aplicó la PC6M y al finalizar se midió signos vitales de presión arterial y saturación de oxígeno. Luego de un descanso de 10 min se realizó el test de Storer y después se midió signos vitales.

Para determinar diferencias entre ambos sexos en datos básicos, signos vitales, rendimiento en la PC6M y test de Storer, se usó la prueba de norma- 
Tabla 1. Características de la muestra

\begin{tabular}{|lccccccc|}
\hline & \multicolumn{2}{c}{ Total $(\mathbf{n}=\mathbf{8 0})$} & \multicolumn{2}{c}{ Hombres $(\mathbf{n}=\mathbf{4 0})$} & \multicolumn{2}{c|}{ Mujeres $(\mathbf{n}=\mathbf{4 0})$} & p valor $^{\mathbf{d}}$ \\
Variables & Media & $\mathbf{D E}$ & Media & DE & Media & DE & \\
\hline Edad (años) & 22,5 & 2 & 22,5 & 1,9 & 22,5 & 1,8 & 0,943 \\
\hline Peso $(\mathrm{kg})$ & 72,7 & 8,7 & 67,3 & 10,1 & 62 & 8,4 & $<0,001$ \\
\hline Estatura $(\mathrm{m})$ & 1,73 & 0,06 & 1,67 & 0,09 & 1,6 & 0,06 & $<0,001$ \\
\hline IMC $\left(\mathrm{kg} / \mathrm{m}^{2}\right)$ & 24,2 & 2,7 & 24,2 & 2,6 & 24,1 & 2,6 & 0,898 \\
\hline PAS reposo $(\mathrm{mmHg})$ & 124 & 13 & 119,1 & 13,9 & 114,2 & 13,1 & 0,001 \\
PAD reposo $(\mathrm{mmHg})$ & 72,3 & 8,4 & 73,6 & 8,3 & 74,8 & 8,2 & 0,191 \\
\hline FC reposo $(\mathrm{p} / \mathrm{m})$ & 71 & 11 & 77 & 12,7 & 82 & 11,9 & $<0,001$ \\
\hline Saturación $\mathrm{O}_{2}(\%)$ & 97,7 & 2 & 97,7 & 1,7 & 97,8 & 2,3 & 0,96 \\
\hline
\end{tabular}

DE: desviación estándar; diferencias entre hombres y mujeres; IMC: índice de masa corporal; PAS: presión arterial sistólica; PAD: presión arterial diastólica; FC: frecuencia cardíaca; $\mathrm{O}_{2}$ : oxígeno.

Tabla 2. Rendimiento en la prueba de caminata de seis minutos

\begin{tabular}{|c|c|c|c|c|c|c|c|}
\hline \multirow[b]{2}{*}{ Variables } & \multicolumn{2}{|c|}{ Total $(n=80)$} & \multicolumn{2}{|c|}{ Hombres $(n=40)$} & \multicolumn{2}{|c|}{ Mujeres $(n=40)$} & \multirow[t]{2}{*}{ p valor } \\
\hline & Media & DE & Media & DE & Media & DE & \\
\hline FC promedio (p/m) & 140,2 & 17,8 & 135,4 & 17,1 & 145,1 & 17,3 & 0,014 \\
\hline FC (\%) & 71 & 8,9 & 68,5 & 8,7 & 73,4 & 8,6 & 0,013 \\
\hline FCr 20 seg & 138,3 & 21,4 & 131,4 & 22,8 & 145,3 & 17,7 & 0,004 \\
\hline $\mathrm{FCr}(\%)$ & 70 & 10,8 & 66,5 & 11,5 & 73,5 & 8,8 & 0,003 \\
\hline PSE & 10,3 & 1,8 & 10,5 & 1,7 & 10,2 & 1,9 & 0,491 \\
\hline Distancia (m) & 669,2 & 52,2 & 696,4 & 48 & 642 & 41,2 & $<0,001$ \\
\hline PAS $(\mathrm{mmHg})$ & 123,1 & 13,6 & 130,4 & 11,9 & 115,9 & 11 & $<0,001$ \\
\hline PAD $(\mathrm{mmHg})$ & 76,6 & 8,1 & 75,5 & 7,9 & 77,6 & 8,2 & 0,235 \\
\hline Saturación $\mathrm{O}_{2}$ (\%) & 98 & 3,2 & 97,6 & 4,4 & 98,5 & 1,2 & 0,226 \\
\hline
\end{tabular}

DE: desviación estándar; 'diferencias entre hombres y mujeres; FC: frecuencia cardíaca; FCr: frecuencia cardíaca de recuperación; FCr (\%): porcentaje de FC máxima a los 20 seg de recuperación; PSE: percepción subjetiva del esfuerzo; PAS: presión arterial sistólica; PAD: presión arterial diastólica; $\mathrm{O}_{2}$ : oxígeno.

lidad de Shapiro-Wilk, el supuesto de homocedasticidad de Levene y se aplicó la prueba t-Student para muestras independientes. La asociación entre el $\mathrm{VO}_{2}$ máx. y el IMC, distancia y frecuencia cardíaca de recuperación (FCr) se estableció con la correlación de Spearman para luego utilizarlas en una regresión multivariada. La bondad de ajuste se evaluó con el coeficiente de determinación $\mathrm{R}^{2} \mathrm{y}$ para la validez del modelo predictivo se determinó los supuestos distribucionales de normalidad de los residuos estandarizados, el supuesto de independencia de los residuos con la prueba de Durbin-Watson, el supuesto de homocedasticidad con la prueba de Levene, y finalmente se verificó concordancia entre lo observado y lo previsto del $\mathrm{VO}_{2}$ máx. con el diagrama de Bland-Altman. La significancia fue de $\mathrm{p}<0,05$ con el programa SPSS versión 22 (IBM, EE.UU.).

\section{Resultados}

En nivel de esfuerzo en la PC6M difiere entre hombres y mujeres en cuanto a la FC, la FCr y la distancia alcanzada, sin embargo, la PSE no es distinta (Tabla 2). 
En la Tabla 3 el rendimiento en el test de $\mathrm{VO}_{2}$ máx. también es distinto entre hombres y mujeres en la potencia alcanzada y el $\mathrm{VO}_{2}$ máx., inclusive en la PSE, pero no en la FC. Al igual que en reposo, después de la caminata y del test de Storer la presión arterial sistólica es estadísticamente distinta entre ambos sexos (Tabla 3).

En la Tabla 4 se aprecia que el sexo, la FCr, la distancia y el IMC explican el 73,1\%, el 17,9\%, el $32,8 \%$ y el $3,4 \%$ de la varianza del $\mathrm{VO}_{2}$ máx., respectivamente.

La ecuación de predicción tiene un valor de $\mathrm{r}=0,95$ y la bondad de ajuste es de $\mathrm{R}^{2}=0,91$.

VO máx. $\left(\mathrm{ml} \cdot \mathrm{min}^{-1}\right)=-3672,585+$ $(966,472 \times$ Sexo $)+(-18,492 \times$ FCr $)+$ $(9,191 \times$ Distancia $)+(87,707 \times$ IMC $)$
Donde el sexo para hombres $=2$ y mujeres $=1$, la FCr a los 20 seg en $\mathrm{p} / \mathrm{m}$, la distancia en metros de la PC6M y el IMC en $\mathrm{kg} / \mathrm{m}^{2}$.

El modelo predictivo es válido, pues los residuos estandarizados cumplieron el supuesto de normalidad ( $\mathrm{p}=0,06971)$, se comprobó el supuesto de independencia $(\mathrm{p}=0,1569)$ y se verificó el supuesto de homocedasticidad ( $\mathrm{p}$ $=0,8313)$. Por último, según Bland-Altman la diferencia de medias entre la PC6M y el test de Storer es $-0,201 \pm 301,2 \mathrm{ml} \cdot \mathrm{min}^{-1}$ (sesgo promedio cercano a cero) con límites de concordancia de $-590,55$ y 590,14 y no hubo diferencias significativas entre el $\mathrm{VO}_{2}$ máx. estimado en la PC6M y el observado en el test de Storer $(p=0,995)$ con intervalo de confianza del $95 \%(-3,344$ a 3,184$)$, que indica que la $\mathrm{PC} 6 \mathrm{M}$ predice el $\mathrm{VO}_{2}$ máx.

Tabla 3. Rendimiento en el test de Storer

\begin{tabular}{|c|c|c|c|c|c|c|c|}
\hline \multirow[b]{2}{*}{ Variables } & \multicolumn{2}{|c|}{ Total $(n=80)$} & \multicolumn{2}{|c|}{ Hombres $(n=40)$} & \multicolumn{2}{|c|}{ Mujeres $(n=40)$} & \multirow[t]{2}{*}{ p valor va $^{\text {d }}$} \\
\hline & Media & DE & Media & DE & Media & DE & \\
\hline Vatios & 285,8 & 74,2 & 343,5 & 55,2 & 228 & 35,7 & $<0,001^{w}$ \\
\hline $\mathrm{FC}(\mathrm{p} / \mathrm{m})$ & 182,9 & 4,1 & 183 & 3,6 & 182,8 & 4,6 & 0,894 \\
\hline FC (\%) & 92,6 & 2 & 92,6 & 1,7 & 92,6 & 2,3 & 0,947 \\
\hline $\mathrm{VO}_{2}$ máx. ml:min ${ }^{-1}$ & 3.489 & 1.001 & $4.355,4$ & 598,6 & $2.622,6$ & 362,5 & $<0,001^{w}$ \\
\hline PSE & 16,2 & 2,2 & 16,7 & 2 & 15,7 & 2,4 & 0,049 \\
\hline PAS (mmHg) & 122,7 & 15,6 & 129 & 16,8 & 116,4 & 11,4 & $<0,001$ \\
\hline PAD $(\mathrm{mmHg})$ & 70 & 9,6 & 68,5 & 10,1 & 71,6 & 9 & 0,148 \\
\hline Saturación $\mathrm{O}_{2}(\%)$ & 98,3 & 0,8 & 97,8 & 0,9 & 98,8 & 0,4 & $<0,001^{w}$ \\
\hline
\end{tabular}

DS: desviación estándar; ddiferencias entre hombres y mujeres; wWilcoxon; FC: frecuencia cardíaca; FC (\%): porcentaje de FC máxima; PSE: percepción subjetiva del esfuerzo; PAS: presión arterial sistólica; PAD: presión arterial diastólica; $\mathrm{O}_{2}$ : oxígeno.

Tabla 4. Predictores univariables y modelo de regresión que explica la varianza del $\mathrm{VO}_{2}$ máx. $(n=80)$

\begin{tabular}{|c|c|c|c|c|c|c|c|c|}
\hline \multirow[b]{2}{*}{ Variables } & \multirow[b]{2}{*}{$\mathbf{R 2}$} & \multirow[b]{2}{*}{ p-valor } & \multicolumn{2}{|c|}{$\begin{array}{l}\text { Coeficientes no } \\
\text { estandarizados }\end{array}$} & \multirow[b]{2}{*}{$\mathbf{t}$} & \multirow[b]{2}{*}{ p-valor } & \multicolumn{2}{|c|}{$\begin{array}{l}95 \% \text { intervalo de } \\
\text { confianza para B }\end{array}$} \\
\hline & & & B & $\begin{array}{c}\text { Error } \\
\text { estándar }\end{array}$ & & & $\begin{array}{l}\text { Límite } \\
\text { inferior }\end{array}$ & $\begin{array}{l}\text { Límite } \\
\text { superior }\end{array}$ \\
\hline Constante & & & $-3672,585$ & 681,584 & $-5,388$ & $<0,001$ & $-5030,672$ & $-2314,498$ \\
\hline Sexo & 0,731 & $<0,001$ & 966,472 & 101,446 & 9,527 & $<0,001$ & 764,336 & 1168,609 \\
\hline $\mathrm{FCr}$ & 0,179 & $<0,001$ & $-18,492$ & 2,073 & $-8,922$ & $<0,001$ & $-22,622$ & $-14,362$ \\
\hline Distancia & 0,328 & $<0,001$ & 9,191 & 0,983 & 9,349 & $<0,001$ & 7,232 & 11,150 \\
\hline IMC & 0,034 & 0,101 & 87,707 & 14,156 & 6,196 & $<0,001$ & 59,502 & 115,913 \\
\hline
\end{tabular}

FCr: frecuencia cardíaca de recuperación en 20 seg; Distancia en metros; IMC: índice de masa corporal en kg/m². 




Figura 1. Diagrama de Bland-Altman, acuerdo entre lo observado y la predicción. Eje X. Promedios del $\mathrm{VO}_{2}$ máx. $\mathrm{ml} \cdot \mathrm{min}^{-1}\left(\mathrm{VO}_{2}\right.$ observado y previsto). Eje $Y$. Diferencias en el del $\mathrm{VO}_{2}$ máx. $\mathrm{ml} \cdot \mathrm{min}^{-1}$ $\left(\mathrm{VO}_{2}\right.$ observado y previsto). entre $-3,344$ y $3,184 \mathrm{ml} \cdot \mathrm{min}^{-1}$. El análisis mostró que dos pares $(2,5 \%)$ del $\mathrm{VO}_{2}$ máx. están fuera de los límites (Figura 1).

\section{Discusión}

El objetivo fue elaborar una ecuación para estimar el $\mathrm{VO}_{2}$ máx. a partir de la PC6M para estudiantes universitarios chilenos de ambos sexos. Según nuestra revisión, este es el primer estudio en Chile que elabora dicha ecuación para universitarios de diferente condición física, exceptuando a estudiantes de educación física. Consideramos que este es el principal aporte al conocimiento respecto a la pertinencia de evaluar con la prueba de caminata. Además, hemos incorporado la frecuencia cardíaca de recuperación para incrementar el poder predictivo, variable que no consideran otras ecuaciones, las que se discuten más adelante, y que también es un hallazgo interesante por su novedad y valor práctico de aplicación. También, evidenciamos que la PC6M puede ser aplicable en estudiantes universitarios, por una parte considerando que es un grupo vulnerable al estar sujetos a diversos cambios que podrían influir sobre sus conductas de vida, entre ellas los hábitos de actividad física, por eso la evaluación a través de la PC6M podría significar un aporte a su salud, y por otra parte la PC6M al ser de baja intensidad se acomoda a los universitarios quienes generalmente realizan poca actividad física o no la realizan desde la educación media, o en algunos casos son sedentarios.

Generalmente la PC6M se aplica en instancias clínicas de tal modo que se ha relacionado con el $\mathrm{VO}_{2}$ en personas con hipertensión pulmonar ${ }^{16,17}$, con parálisis cerebral ${ }^{18}$, con hemiparesia crónica ${ }^{19}$, con discapacidad intelectual ${ }^{20}$ y con enfermedad pulmonar obstructiva crónica ${ }^{21}$. Solo en algunos casos en personas saludables ${ }^{8,22} \mathrm{y}$ en estudiantes de educación física ${ }^{23}$ y aunque estas últimas investigaciones establecen variables potencialmente predictoras no elaboran ecuaciones de estimación del $\mathrm{VO}_{2}$ máx., por eso es relevante generar un modelo predictivo para los universitarios, etapa clave para la evaluación de la condición física asociada a morbilidad y mortalidad en décadas posteriores ${ }^{24}$.

Por otra parte, el número de participantes de esta investigación fue 40 hombres y 40 mujeres lo que está referenciado por algunos estudios sobre la cantidad de participantes para generar un modelo predictivo. Se sostiene que las ecuaciones deben utilizarse con precaución cuando se han elaborado con pocos individuos ${ }^{10}$, aunque tengan significancia estadística ${ }^{25}$, esta idea es respaldada en una investigación con 35 participantes ${ }^{26}$ aunque también encontramos un modelo predictivo del 
$\mathrm{VO}_{2}$ máx. para la PC6M con 12 personas ${ }^{10}$. Otros estudios consideran muestras mayores de 61 personas para investigar sobre la $\mathrm{PC}^{2} \mathrm{M}^{20}$ y también se ha propuesto una ecuación para predecir la tasa máxima de trabajo en una prueba cardiopulmonar con 53 participantes ${ }^{27}$.

Las fórmulas para predecir el $\mathrm{VO}_{2}$ máx. están en la Tabla 5. El presente estudio tiene la correlación más alta comparado con otros trabajos, inclusive alguno tiene un valor moderado y dos investigaciones no lo reportan.

La mayoría de las ecuaciones se elaboraron para edades adultas, cuatro de estos modelos para personas con patologías y llama la atención que una investigación tiene muy pocos participantes. Nuestra investigación tiene una muestra promedio respecto a las otras cinco investigaciones de la Tabla 5, generada con hombres y mujeres al igual que en la mayoría de los estudios. Se aprecia que la cinta rodante y el $\mathrm{VO}_{2}$ en $\mathrm{ml} \cdot \mathrm{kg} \cdot \mathrm{min}^{-1}$ predomi- na en las investigaciones, lo que no ocurrió con los universitarios chilenos, también que la mitad de los casos se presenta como $\mathrm{VO}_{2}$ peak dando a entender que fue el valor más alto del $\mathrm{VO}_{2}$ en la última etapa del test de criterio y que no se alcanzó una meseta en la curva pese al aumento de la intensidad para determinar el $\mathrm{VO}_{2}$ máx.

Las principales variables medidas en la PC6M son la distancia, edad, peso corporal, estatura, IMC, variables antropométricas y el sexo ${ }^{28} \mathrm{de}$ manera que casi en su totalidad concuerdan con las que medimos en los universitarios y con las que aparecen en los estudios de la tabla 5, además en las ecuaciones existen diferentes variables pero la que es común para todos es la distancia recorrida. Para esta variable ${ }^{29}$ se reportan $702 \pm 54$ y $736 \pm 79$ metros en mujeres y hombres universitarios, respectivamente, también ${ }^{30} 643,5 \pm 63,1$ metros para hombres jóvenes saludables, y personas chilenas de ambos sexos de 20 a 29 años deberían realizar entre

Tabla 5. Ecuaciones que predicen el $\mathrm{VO}_{2}$ en la prueba de caminata de seis minutos

\begin{tabular}{|c|c|c|c|c|c|c|c|c|}
\hline Autor & $\mathbf{n}$ & Sexo & Edad & $\begin{array}{l}\text { Caracte- } \\
\text { rística }\end{array}$ & $\begin{array}{c}\text { Test criterio } \\
\mathrm{VO}_{2}\end{array}$ & Ecuación & $\mathbf{r}$ & p valor \\
\hline PE & 80 & $\mathrm{H}$ y M & $22,5 \pm 2$ & $\begin{array}{l}\text { Universita- } \\
\quad \text { rios }\end{array}$ & $\begin{array}{l}\text { Cicloergómetro } \\
\text { (i)* máx. }\end{array}$ & $\begin{array}{c}-3672,585+(966,472 \times \text { sexo } \\
[1: \text { mujer, } 2: \text { hombre }])+ \\
(-18,492 \times \text { FCr } 20 \text { seg })+(9,191 \\
\times \text { distancia })+(87,707 \times \text { IMC })\end{array}$ & 0,95 & $<0,001$ \\
\hline (5) & 81 & Hy M & $48,6 \pm 8,1$ & $\begin{array}{l}\text { Enfer- } \\
\text { medad } \\
\text { cardíaca }\end{array}$ & $\begin{array}{l}\text { Cinta rodante } \\
(\text { i })^{* *} \text { peak }\end{array}$ & $\begin{array}{c}53,43+(1,35 \times \text { sexo }[0: \text { mujer } \\
\text { 1: hombre }])-(5,59 \times \text { NYHA }) \\
+(0,01 \times \text { distancia })-(0,29 \times \\
\quad \text { edad })-(0,035 \times \text { IMC })\end{array}$ & 0,81 & $<0,001$ \\
\hline (9) & 97 & $\mathrm{H}$ y $\mathrm{M}$ & $69,1 \pm 5,6$ & $I C S-I C D$ & $\begin{array}{l}\text { Cicloergómetro } \\
(\mathrm{d})^{* *} \text { peak }\end{array}$ & $0,01426 \times$ distancia $(m)+7,222$ & 0,54 & $<0,001$ \\
\hline (7) & 97 & $\mathrm{H}$ y $\mathrm{M}$ & $13 \pm 2,9$ & Obesos & $\begin{array}{l}\text { Cinta rodante } \\
(\mathrm{d})^{* *} \text { máx. }\end{array}$ & $\begin{aligned} 26,9+0,014 & \times \text { distancia }-0,38 \\
& \times \mathrm{IMC}\end{aligned}$ & $\mathrm{nr}$ & $\mathrm{nr}$ \\
\hline$(10)$ & 12 & $\mathrm{H}$ y $M$ & $60-70$ & Saludables & $\begin{array}{l}\text { Cinta rodante } \\
(\mathrm{d})^{*} \text { máx. }\end{array}$ & $\begin{array}{c}2830,6-(45,2 \times \text { edad años }) \\
+(4,7 \times \text { peso } \mathrm{kg})+(12,3 \times \\
\text { estatura } \mathrm{cm})+(1,75 \times \text { distancia } \\
\mathrm{m})+\left(0,309 \times \mathrm{VO}_{2} \text { en PC6M }\right)- \\
(12,4 \times \mathrm{FC} \mathrm{p} / \mathrm{m})\end{array}$ & 0,97 & $<0,01$ \\
\hline$(32)$ & 77 & $\mathrm{H}$ y $\mathrm{M}$ & $65 \pm 11$ & Saludables & $\begin{array}{l}\mathrm{PC}_{\mathrm{MM} \mathrm{VO}} \\
(\mathrm{d})^{* *}\end{array}$ & $-14,1+(0,058 \times$ distancia $)$ & 0,8 & $<0,001$ \\
\hline$(11)$ & 120 & $\mathrm{H}$ & $68,1 \pm 12,8$ & DSVI & $\begin{array}{l}\text { Cinta rodante } \\
(\mathrm{d})^{* *} \text { peak }\end{array}$ & $\begin{array}{c}11,92+(1,48 \times \text { VEF })+(1,12 \times \\
H b g / d l)+(0,016 \times \text { distancia } m) \\
-(0,33 \times I M C)-(0,11 \times \text { edad })\end{array}$ & $\mathrm{nr}$ & $<0,05$ \\
\hline
\end{tabular}

PE: presente estudio; H: hombre; M: mujer; ICS: insuficiencia cardíaca sistólica; ICD: insuficiencia cardíaca diastólica; DSVI: disfunción sistólica ventricular izquierda; (i): medición indirecta; (d): medición directa; ${ }^{*} \mathrm{ml}^{*} \mathrm{~kg} \cdot \mathrm{min}^{-1} ;{ }^{*} \mathrm{ml} \cdot \mathrm{min}-1 ;$ peak: $\mathrm{VO}_{2}$ peak; máx.: VO $\mathrm{V}_{2}$ máx.; NYHA: Clasificación funcional de la Asociación del Corazón, Nueva York-EEUU; VEF: volumen espiratorio forzado; FCr: frecuencia cardíaca de recuperación; nr: no reportado. 
600 y 800 metros $^{31}$. En nuestro estudio encontramos distancias de 669,2 $\pm 52,2$, de $696,4 \pm 48$ y $642 \pm 41,2$ metros en la muestra, en hombres y mujeres, respectivamente, lo que se asemeja con los dos últimos estudios citados.

Por su parte el IMC explica en algún porcentaje el $\mathrm{VO}_{2}$ en tres investigaciones y también en esta investigación $\left(\mathrm{R}^{2}=0,034\right)$. El sexo es parte del modelo predictivo en nuestro estudio y en el estudio de Costa ${ }^{5}$, pero la investigación de Ingle ${ }^{11}$ no lo incluye ya que se realizó sólo con hombres, y la edad aparece en tres ecuaciones de la tabla 5. Las variables que se asocian al $\mathrm{VO}_{2}$ máx. deben ser, de fácil medición para hacer una transferencia desde el laboratorio a la evaluación en terreno y no considerar instrumental de difícil acceso como en la ecuación de Ingle ${ }^{11}$ donde se mide el volumen espiratorio forzado y la hemoglobina, sin desconocer que son importantes y que el modelo es adecuado.

La FCr medida después de la PC6M tiene relación con el $\mathrm{VO}_{2}$ máx. por eso que la incluimos en el presente estudio. Esta asociación es inversa ${ }^{33,34} y$ se ha obtenido en estudios que utilizan solamente la FC en los primeros 30 segundos de recuperación de una prueba submáxima para predecir el $\mathrm{VO}_{2}$ máx $^{35-39}$. La utilidad práctica para medir la FCr es que no se interrumpe el test y se puede medir de forma estacionaria con mayor control.

La edad de los universitarios (22,5 \pm 2 años) no se consideró en el modelo regresivo pero algunas investigaciones sugieren incluirlo ${ }^{20} \mathrm{y}$ algunas fórmulas que predicen el $\mathrm{VO}_{2}$ máx. en la PC6M la incorporan ${ }^{5,10,11}$ lo que es una potencial limitante para este estudio ya que el $\mathrm{VO}_{2}$ tiene edades de mayor desarrollo y en otras que disminuye. Sin embargo, la edad mostró una correlación muy baja y no significativa con el $\mathrm{VO}_{2}$ máx. $(\mathrm{r}=0,022$; $\mathrm{p}=0,848)$.

Como limitación y a la vez como línea de futuro, se realizará la medición directa del $\mathrm{VO}_{2}$ máx., en cicloergómetro o cinta rodante. En este sentido, ahondar en nuevos modelos para estimar el $\mathrm{VO}_{2}$ máx. en grupos poco estudiados como los universitarios, es necesario por las implicancias prácticas que puede significar para los profesionales de la salud y la actividad física encontrar en una prueba de campo, sencilla, fiable y fácil de medir valores similares a las pruebas de laboratorio.

Se concluye que La PC6M puede predecir el $\mathrm{VO}_{2}$ máx. de los hombres y mujeres universitarios ya que las variables de distancia, IMC, sexo y FCr lo explican en un alto porcentaje, con una alta correlación y significancia estadística. El IMC tiene menor relación con el $\mathrm{VO}_{2}$ máx., la distancia y la FCr se asocian de manera moderada y esta última de manera inversa.

Agradecimientos: Se agradece a los estudiantes universitarios que realizaron las pruebas y a los académicos que colaboraron en la elaboración del estudio.

\section{Referencias}

1. Morris NR, Seale H, Harris J, Hall K, Lin ACW, Kermeen F. Gas exchange responses during 6-min walk test in patients with pulmonary arterial hypertension. Respirology 2017; 22 (1): 165-71.

2. Zapico AG, Fuentes D, Rojo-Tirado MA, Calderón FJ, Rosenzweig EB, Garofano RP. Predicting peak oxygen uptake from the 6-minute walk test in patients with pulmonary hypertension. J Cardiopulm Rehabil Prev 2016; 36 (3): 203-8.

3. Fleg JL, Pina IL, Balady GJ, Chaitman BR, Fletcher B, Lavie C, et al. Assessment of functional capacity in clinical and research applications - An advisory from the Committee on Exercise, Rehabilitation, and Prevention, Council on Clinical Cardiology, American Heart Association. Circulation 2000; 102 (13): 1591-7.

4. Harmsen WJ, Ribbers GM, Slaman J, Heijenbrok-Kal $\mathrm{MH}$, Khajeh L, van Kooten F, et al. The six-minute walk test predicts cardiorespiratory fitness in individuals with aneurysmal subarachnoid hemorrhage. Top Stroke Rehabil 2016; 24 (4): 250-5.

5. Costa HS, Lima MMO, Alencar MCN, Sousa GR, Figueiredo PHS, Nunes MCP, et al. Prediction of peak oxygen uptake in patients with Chagas heart disease: Value of the Six-minute Walk Test. Int J Cardiol 2017; 228 (1): 385-7.

6. Zhang Q, Lu H, Pan S, Lin Y, Zhou K, Wang L. 6MWT performance and its correlations with VO2 and handgrip strength in home-dwelling mid-aged and older Chinese. Int J Environ Res Public Health 2017; 14 (5): 473-82.

7. Vanhelst J, Fardy PS, Salleron J, Beghin L. The six-minute walk test in obese youth: reproducibility, validity, and prediction equation to assess aerobic power. Disabil Rehabil 2013; 35 (6): 479-82.

8. Andersson EA, Lundahl G, Wecke L, Lindblom I, Nilsson J. Maximal aerobic power versus performance 
in two aerobic endurance tests among young and old adults. Gerontology 2011; 57 (6): 502-12.

9. Maldonado-Martin S, Brubaker PH, Kaminsky LA, Moore JB, Stewart KP, Kitzman DW. The relationship of 6-min walk to VO2peak and VT in older heart failure patients. Med Sci Sport Exer 2006; 38 (6): 1047-53.

10. Kervio G, Carre F, Ville NS. Reliability and intensity of the six-minute walk test in healthy elderly subjects. Med Sci Sport Exer 2003; 35 (1): 169-74.

11. Ingle L, Goode K, Rigby ASR, Cleland JGF, Clark AL. Predicting peak oxygen uptake from 6-min walk test performance in male patients with left ventricular systolic dysfunction. Eur J Heart Fail 2006; 8 (2): 198-202.

12. Vásquez-Gómez J, Castillo-Retamal M, Ramírez-Campillo R. A six-minute walking test: maximum oxygen consumption in physical education students. J Phys Educ 2018; 29 (1): e2919.

13. Gutiérrez Clavería M, Beroíza WT, Cartagena SC, Caviedes I, Céspedes J, Gutiérrez Navas N, et al. Prueba de caminata de seis minutos. Rev Chil Enf Respir 2009; 25 (1): 15-24.

14. Borg GAV, Kaijser L. A comparison between three rating scales for perceived exertion and two different work tests. Scand J Med Sci Sports 2006; 16 (1): 57-69.

15. Storer TW, Davis JA, Caiozzo VJ. Accurate prediction of - VO2max in cycle ergometry. Med Sci Sports Exerc 1990; 22 (5): 704-12.

16. Acar S, Savci S, Kardibak D, Kahraman BO, Akdeniz B, Ozpelit E, et al. Clinical correlation between the 6-min walk test and cardiopulmonary exercise testing in patients with pulmonary arterial hypertension. Turkish J Med Sci 2016; 46 (6): 1658-64.

17. Neal JE, Lee AS, Burger CD. Submaximal exercise testing may be superior to the 6-min walk test in assessing pulmonary arterial hypertension disease severity. Clin Respir J 2014; 8 (4): 404-9.

18. Slaman J, Dallmeijer A, Stam H, Russchen H, Roebroeck $\mathrm{M}$, van den Berg-Emons $\mathrm{R}$, et al. The six-minute walk test cannot predict peak cardiopulmonary fitness in ambulatory adolescents and young adults with cerebral palsy. Arch Phys Med Rehab 2013; 94 (11): 2227-33.

19. Ovando AC, Michaelsen SM, Carvalho TD, Herber, V. Evaluation of cardiopulmonary fitness in individuals with hemiparesis after cerebrovascular accident. Arq Bras Cardiol 2011; 96 (2): 140-7.

20. Elmahgoub SS, Van de Velde A, Peersman W, Cambier D, Calders P. Reproducibility, validity and predictors of six-minute walk test in overweight and obese adolescents with intellectual disability. Disabil Rehabil 2012; 34 (10): 846-51.

21. Turner SE, Eastwood PR, Cecins NM, Hillman DR,
Jenkins SC. Physiologic responses to incremental and self-paced exercise in COPD: a comparison of three tests. Chest 2017; 126 (3): 766-73.

22. Limsuwan A, Wongwandee R, Khowsathit P. Correlation between 6-min walk test and exercise stress test in healthy children. Acta Paediatr 2010; 99 (3): 438-41.

23. Andersson EA, Nilsson J. Can a six-minute shuttle walk test predict maximal oxygen uptake? Gazz Med Ital 2011; 170 (3): 163-70.

24. Sesso HD, Paffenbarger RS, Lee IM. Physical activity and coronary heart disease in men. Circulation 2000; 102 (9): 975-80.

25. Tseng BY, Kluding P. The relationship between fatigue, aerobic fitness, and motor control in people with chronic stroke: a pilot study. J Geriatr Phys Ther 2009; 32 (3): 97-102.

26. Doutreleau SDMP, Talha S, Charloux A, Piquard F, Geny B. Can the six-minute walk test predict peak oxygen uptake in men with heart transplant? Arch Phys Med Rehabil 2009; 90 (1): 51-7.

27. Cavalheri V, Hernandes NA, Camillo CA, Probst VS, Ramos D, Pitta F. Estimation of maximal work rate based on the 6-minute walk test and fat-free mass in chronic obstructive pulmonary disease. Arch Phys Med Rehab 2010; 91 (10): 1626-8.

28. Dourado VZ. Ecuaciones de referencia para el test de caminata de seis minutos en individuos sanos. Arq Bras Cardiol 2011; 96 (6): 128-38.

29. Grindrod D, Paton CD, Knez WL, O’Brien BJ. Six minute walk distance is greater when performed in a group than alone. Br J Sports Med 2006; 40 (10): 876-7.

30. Davi SF, Arcuri JF, Labadessa IG, Pessoa BV, Costa JN, Sentanin AC. Reprodutibilidade do teste de caminhada e do degrau de 6 minutos em adultos jovens saudáveis. Rev Bras Med Esporte 2014; 20 (3): 214-8.

31. Osses R, Yáñez J, Barría P, Palacios S, Dreyse J, Díaz $\mathrm{O}$, et al. Prueba de caminata en seis minutos en sujetos chilenos sanos de 20 a 80 años. Rev Med Chile 2010; 138 (9): 1124-30.

32. Hill K, Wickerson LM, Woon LJ, Abady AH, Overend TJ, Goldstein RS, et al. The 6-min walk test: responses in healthy Canadians aged 45 to 85 years. Appl Physiol Nutr Metab 2011; 36 (5): 643-9.

33. Francis K, Culpepper M. Height-adjusted, rate-specific, single-stage step test for predicting maximal oxygen consumption. South Med J 1989; 82 (5): 602-6.

34. Dimkpa U. Post-exercise heart rate recovery: an index of cardiovascular fitness. J Exerc Physiol Online 2009; 12 (2): 10-22.

35. McArdle WD, Katch FI, Pechar GS, Jacobson L, Ruck S. Reability and interrelationships between maximal oxy- 
gen intake, physical work capacity and step-test scores in college women. Med Sci Sports Exerc 1972; 4 (4): 182-6.

36. Francis K, Feinstein R. A simple height-specific and rate-specific step test for children. South Med J 1991; 84 (2): 169-74.

37. Santo AS, Golding LA. Predicting maximum oxygen uptake from a modified 3-minute step test. Res Q Exerc Sport 2003; 74 (1): 110-5.
38. Chatterjee S, Chatterjee P, Mukherjee PS, Bandyopadhyay A. Validity of Queen's College step test for use with young Indian men. Br J Sports Med 2004; 38 (3): 28991.

39. Chatterjee S, Chatterjee P, Bandyopadhyay A. Validity of Queen's College Step Test for estimation of maximum oxygen uptake in female students. Indian J Med Res 2005; 121 (1): 32-5. 